\title{
Counterpropagating beams in biased photorefractive crystals: Anisotropic theory
}

\author{
K. Motzek, ${ }^{1}$ M. Belić, ${ }^{2,3}$ T. Richter, ${ }^{1}$ C. Denz ${ }^{4}$ A. Desyatnikov, ${ }^{4,5}$ Ph. Jander, ${ }^{4}$ and F. Kaiser ${ }^{1}$ \\ ${ }^{1}$ Institute of Applied Physics, Darmstadt University of Technology, 64289 Darmstadt, Germany \\ ${ }^{2}$ Texas A\&M University at Qatar, P. O. Box 5825, Doha, Qatar \\ ${ }^{3}$ Institute of Physics, P. O. Box 57, Belgrade, Serbia \\ ${ }^{4}$ Institute of Applied Physics, Westfälische Wilhelms-Universität Münster, 48149 Münster, Germany \\ ${ }^{5}$ Nonlinear Physics Centre, Research School of Physical Sciences and Engineering, Australian National University, \\ Canberra, Australian Capital Territory 0200, Australia
}

(Received 25 July 2004; published 19 January 2005)

\begin{abstract}
We formulate an anisotropic nonlocal theory of the space charge field induced by the coherent counterpropagating beams in biased photorefractive crystals. We establish that the competition between the drift and diffusion terms has to be taken into account when the crystal $\hat{c}$ axis is tilted with respect to the propagation direction of the beams. We demonstrate that this configuration combines the features of both spatial soliton formation without energy exchange and two-wave mixing with energy exchange leading to pattern formation.
\end{abstract}

DOI: 10.1103/PhysRevE.71.016610

PACS number(s): 42.65.Tg, 42.65.Jx

Coherent interaction of counterpropagating (CP) light beams in Kerr-type and photorefractive (PR) media has been treated in a number of papers [1-7], using mainly an isotropic and local approximation to the nonlinear response of the medium. This means that the change in the refractive index, caused by light, is spatially isotropic and depends locally on the light intensity. It has been shown, however, that the agreement between theoretical predictions and experimental results in PR crystals can be improved by using an anisotropic nonlocal model for the nonlinearity [8]. The change in the refractive index at one point then depends on the intensity distribution at other points, and it includes a directional dependence on the applied electric field, which is necessary for the screening effect. Still, an exact isotropic local theory was formulated only for the one-transverse-dimensional (1D) copropagating beams in PR crystals [9].

Here we formulate an anisotropic nonlocal theory of the space charge field induced by the coherent counterpropagating beams in biased PR crystals. We show that the anisotropic nonlocal theory yields significantly different results from the isotropic local model, especially when the crystal $\hat{c}$ axis is tilted with respect to the direction of propagation of the beams. We demonstrate that a more complete description of counterpropagating beams requires inclusion of both the drift and diffusion terms.

We assume that the optical electric field is given as the sum of slowly varying amplitudes $F \exp (i k z)+B \exp (-i k z)$ + c.c., $k$ being the wave vector in the crystal, and $F$ and $B$ the envelopes of the beams counterpropagating along the $z$ axis. The light intensity, after averaging in time, builds an interference pattern of the form

$$
I=I_{0}+\varepsilon\left[F B^{*} \exp (2 i k z)+\text { c.c. }\right],
$$

where $I_{0}=|F|^{2}+|B|^{2}$ is the homogeneous light intensity and $\varepsilon$ is the degree of beam coherence $(\varepsilon=1$ for fully coherent and
0 for fully incoherent beams). This pattern modulates the space charge field in the crystal and generates a reflectiontype grating in the index of refraction. Our aim is to evaluate the strength of this grating and its phase shift relative to the intensity interference pattern, and to investigate its influence on the propagation of beams. We expect both the strength and the phase to depend on the angle $\alpha$ between the beams' direction of propagation and the crystal $\hat{c}$ axis.

We choose the $z$ axis to be parallel to the beams' direction of propagation. To utilize the largest component of the electro-optic tensor for soliton formation, the beams' polarization is chosen to lie in the plane of the $\hat{c}$ axis and the $z$ axis. The external electric field is applied parallel to the $\hat{c}$ axis. Hence, we assume that the beams in the transverse plane are localized in the $x$ direction and that the space charge field is confined to the $x-z$ plane (1D geometry). It presents no difficulty to extend our results to two transverse dimensions. Figure 1 depicts the geometry of the problem.

For incoherent beams $(\varepsilon=0)$ the space charge field consists only of a homogeneous component $\mathbf{E}_{s c}(\mathbf{r})=\mathbf{E}_{0}(\mathbf{r})$, where $\mathbf{r}=x \mathbf{x}+z \mathbf{z}$, and $\mathbf{x}$ and $\mathbf{z}$ are the unit vectors. For coherent beams the interference pattern induces an additional modulation of the electric potential $\phi$ generated by the separated space charges in the medium, proportional to $\varepsilon$ :

$$
\phi(\mathbf{r})=\phi_{0}(\mathbf{r})+(\varepsilon / 2)\left[\phi_{+}(\mathbf{r}) \exp (i 2 k z)+\text { c.c. }\right] .
$$

Here the fast and slow oscillations in the $z$ direction are separated in the leading order, by introducing the slowly varying envelope $\phi_{+}$of the potential, with $\left|\partial_{z} \phi_{+}(\mathbf{r})\right|$ $\ll 2 k\left|\phi_{+}(\mathbf{r})\right|$. The potential $\phi$ generates the space charge field, which also consists of a modulated and an unmodulated part: $\mathbf{E}_{s c}=\mathbf{E}_{0}+\mathbf{E}_{+}[\exp (i 2 k z)+$ c.c. $]$. Thus we have $\mathbf{E}_{0}=\boldsymbol{\nabla} \phi_{0}$ and $\mathbf{E}_{+}=\mathbf{x} \mathcal{E}_{x}+\mathbf{z} \mathcal{E}_{z}=\mathbf{x} \partial_{x} \phi_{+}+\mathbf{z} \varepsilon k i \phi_{+}$, where $\boldsymbol{\nabla}=\mathbf{x} \partial_{x}+\mathbf{z} \partial_{z}$.

The charge distribution inside a PR crystal is modeled by 


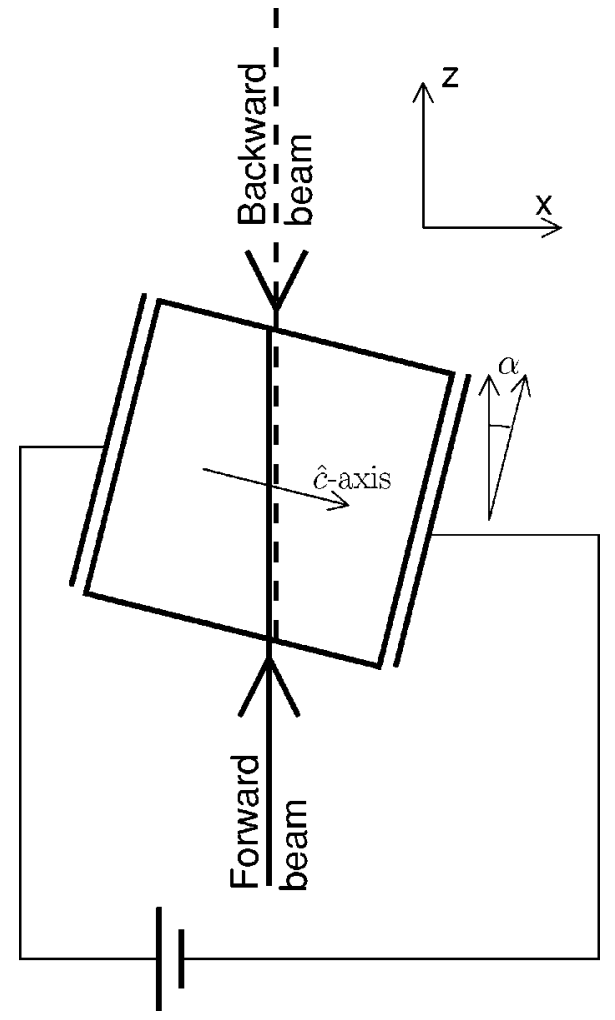

FIG. 1. Geometry of beam propagation in a tilted biased photorefractive crystal.

the Kukhtarev equations [10]. These equations can be reduced to the following potential equation:

$$
\begin{aligned}
& \frac{\tau}{1+I_{0}} \partial_{t}\left(\nabla^{2} \phi\right)+\nabla^{2} \phi+\nabla \ln (1+I) \nabla \phi \\
& =\mathbf{E}_{e} \cdot \nabla \ln (1+I)-\frac{k_{B} T}{q}\left\{\nabla^{2} \ln (1+I)+[\nabla \ln (1+I)]^{2}\right\},
\end{aligned}
$$

using a few well-justified approximations [11]. Here $\tau$ is the relaxation time of the crystal, $\mathbf{E}_{e}$ is the external biasing field, $k_{B}$ is the Boltzmann constant, $T$ is the temperature, and $q$ is the elementary charge. The background illumination, also necessary for the formation of spatial solitons, is now added to the total light intensity.

In the case $\varepsilon=0$ the potential can be calculated just as in the well-known case of incoherent copropagating light beams. Substituting Eq. (2) into Eq. (3) and setting $\varepsilon=0$ yields

$$
\tau \partial_{t} \partial_{x}^{2} \phi_{0}+\left(1+I_{0}\right) \partial_{x}^{2} \phi_{0}+\partial_{x} I_{0} \partial_{x} \phi_{0}=E_{e}^{x} \partial_{x} I_{0}-\kappa \partial_{x}^{2} I_{0},
$$

where $E_{e}^{x}$ is the $x$ component of $\mathbf{E}_{e}$ and $\kappa=k_{B} T / q$ is the diffusion potential. Here we have neglected the derivatives of $\phi_{0}$ and $I_{0}$ with respect to $z$, because the beams typically used in experimental setups have a diameter of about $10 \mu \mathrm{m}$, i.e., $\phi_{0}$ and $I_{0}$ vary in the $x$ direction on a length scale of $10 \mu \mathrm{m}$, whereas in the $z$ direction they vary on the length scale of the diffraction length $L_{D} \approx 2 \mathrm{~mm}$. Therefore, in steady state, i.e., when setting $\partial_{t} \phi_{0}=0$, one obtains the wellknown result

$$
\mathbf{E}_{0}=-\mathbf{x}\left[E_{e}^{x} I_{0}+\kappa \partial_{x} I_{0}\right] /\left(1+I_{0}\right) .
$$

Note that we have chosen the coordinate system such that the $z$ direction is always parallel to the direction of propagation of the beams. Hence $\mathbf{E}_{0}$ is parallel to the $x$ direction, because variations of the light intensity in the $z$ direction are small and hence both drift and diffusion terms in the $z$ direction can be neglected. Under normal experimental conditions $\kappa$ $<0.05 x_{0}\left|\mathbf{E}_{e}\right|$, where $x_{0}=10 \mu \mathrm{m}$ is the beam size, which means that $\mathbf{E}_{0}$ is well approximated by $\mathbf{E}_{0}=-\mathbf{x} E_{e}^{x} I_{0} /\left(1+I_{0}\right)$, i.e., for room temperature and $\varepsilon=0$ diffusion of the charge carriers plays only a minor role. Hence, in that case a local isotropic solution to the space charge field is obtained. However, it has been shown that the term $\kappa \partial_{x} I_{0} /\left(1+I_{0}\right)$, which causes self-bending of the beams, can be of crucial importance for counterpropagating beams [12], particularly for longer propagation lengths, if $\alpha \leqslant 5^{\circ}$.

In the case of mutually coherent $\mathrm{CP}$ beams, i.e., $\varepsilon>0$, substituting expression (2) into Eq. (3), separating the slow and fast oscillating parts, and keeping the terms up to the first order in $\varepsilon$, one finds that $\mathbf{E}_{0}$ is again given by Eq. (3), and that $\phi_{+}$solves

$$
\begin{aligned}
\tau \partial_{t}\left(\partial_{x}^{2} \phi_{+} / 2-2 k^{2} \phi_{+}\right)+\left(1+I_{0}\right) m \partial_{x}^{2} \phi_{0} / 2+\left(1+I_{0}\right) \\
\quad \times\left(\partial_{x}^{2} \phi_{+} / 2-2 k^{2} \phi_{+}\right)+\partial_{x}\left[\left(1+I_{0}\right) m / 2\right] \partial_{x} \phi_{0}+\partial_{x} I_{0} \partial_{x} \phi_{+} \\
=E_{e}^{x} \partial_{x}\left[\left(1+I_{0}\right) m\right]+i k E_{e}^{z}\left(1+I_{0}\right) m \\
\quad-\kappa\left\{\partial_{x}^{2}\left[\left(1+I_{0}\right) m\right] / 4-2 k^{2}\left(1+I_{0}\right) m\right\},
\end{aligned}
$$

where $m=2 F B^{*} /\left(1+I_{0}\right)$ is the modulation depth. This fairly complicated equation can be simplified by noticing that it contains terms of different orders of magnitude. On the one hand there are the terms proportional to $k^{2}=4 \pi / \lambda^{2}$, where $\lambda$ is the wavelength in the medium, typically around $200 \mathrm{~nm}$. On the other hand there are the terms containing $\partial_{x}$ derivatives. Since $\phi, I_{0}$, and $m$ vary in the $x$ direction on the length scale of the size of the beam, i.e., $10 \mu \mathrm{m}$, their partial derivatives can be neglected. Therefore, only the terms proportional to $k^{2}$ and the term $i k E_{e}^{z}\left(1+I_{0}\right) m$ need to be taken into account. Thus we get

$$
2 k^{2} \tau \partial_{t} \phi_{+}+2 k^{2}\left(1+I_{0}\right) \phi_{+}=-2 k^{2} \kappa\left(1+I_{0}\right) m-i k E_{e}^{z}\left(1+I_{0}\right) m .
$$

The steady state solution is $\phi_{+}=-\left[\kappa+i E_{e}^{z} /(2 k)\right] m$; hence

$$
\mathcal{E}_{x}=-\left[\kappa+i E_{e}^{z} /(2 k)\right] \partial_{x} m
$$

and

$$
\mathcal{E}_{z}=-\varepsilon\left(i k \kappa-E_{e}^{z} / 2\right) m .
$$

Even for the values of $E_{e}^{z}$ as high as several $\mathrm{kV} / \mathrm{cm}, k \kappa$ is bigger than $E_{e}^{z}$, which means that the modulated part of the field in the $z$ direction, $\mathcal{E}_{z}$, is diffusion dominated.

With Eqs. (5), (8), and (9) we have a closed set of equations that gives us the space charge field inside the crystal to a good approximation. It remains to find out how the space charge field changes the refractive index and thus influences 
the propagation of beams. The fact that the (birefringent) crystal is tilted with respect to the direction of propagation of the beams has to be taken into account. Linear optics gives us the effective refractive index of the crystal:

$$
n_{\text {eff }}^{2}=\frac{n_{33}^{2} n_{11}^{2}-n_{13}^{4}}{n_{33}^{2} \sin ^{2} \alpha+n_{11}^{2} \cos ^{2} \alpha+2 n_{13}^{2} \sin \alpha \cos \alpha},
$$

where $\varepsilon_{0}\left\{n_{i j}^{2}\right\}$ is the dielectric tensor. $n_{33}$ is the index of refraction for the beams polarized parallel to the crystal $\hat{c}$ axis and $n_{11}$ is the index for the beams polarized perpendicular to $\hat{c} . \alpha$, as mentioned above, is the angle by which the crystal is tilted with respect to the direction of propagation of the beams (see Fig. 1). To show how the modulated and unmodulated parts of the space charge field influence $n_{\text {eff }}$ we take the SBN:75 crystal as an example. The generalization to other photorefractive crystals is straightforward.

In SBN:75 the refractive index can be influenced by the three components of the electro-optic tensor: $r_{33}$ $=1340 \mathrm{pm} / \mathrm{V}, r_{13}=67 \mathrm{pm} / \mathrm{V}$, and $r_{42}=42 \mathrm{pm} / \mathrm{V}$. The values are taken from Ref. [10] and are valid for the light of vacuum wavelength $633 \mathrm{~nm}$. Let us now decompose the nonlinear refractive index change $\delta n^{2}$ into a modulated and unmodulated part: $\delta n^{2}=\delta n_{0}^{2}+\delta n_{m}^{2}[\exp (2 i k z)+\exp (-2 i k z)] / 2$. The space charge field influences the refractive index as follows:

$$
\begin{gathered}
\delta n_{0}^{2}=\mathbf{E}_{0} \cdot \mathbf{x}\left[\left(r_{33} \widetilde{n}_{33}^{4} a_{33}+r_{13} \widetilde{n}_{11}^{4} a_{11}\right) \cos \alpha\right. \\
\left.-r_{42} \widetilde{n}_{33}^{2} \widetilde{n}_{11}^{2} a_{13} \sin \alpha\right], \\
\delta n_{m}^{2}=\left(\mathcal{E}_{x} \cos \alpha+\mathcal{E}_{z} \sin \alpha\right)\left(r_{33} \widetilde{n}_{33}^{4} a_{33}+r_{13} \widetilde{n}_{11}^{4} a_{11}\right) \\
+\left(\mathcal{E}_{x} \sin \alpha+\mathcal{E}_{z} \cos \alpha\right) r_{42} \widetilde{n}_{33}^{2} \widetilde{n}_{11}^{2} a_{13},
\end{gathered}
$$

where $\varepsilon_{0}\left\{\tilde{n}_{i j}\right\}$ is the unperturbed dielectric tensor $a_{i j}$ $=\partial n_{\text {eff }}^{2} / \partial n_{i j}^{2}$, and $\mathbf{E}_{0}$ is given by Eq. (5).

The propagation equations of the beam envelopes in the paraxial approximation are then given by

$$
\begin{gathered}
i \partial_{z} F+\frac{1}{2} \partial_{x}^{2} F=\delta n_{0}^{2} F+\frac{1}{2} \delta n_{m}^{2} B, \\
-i \partial_{z} B+\frac{1}{2} \partial_{x}^{2} B=\delta n_{0}^{2} B+\frac{1}{2}\left(\delta n_{m}^{2}\right)^{*} F,
\end{gathered}
$$

where we have neglected the terms proportional to $\partial \delta n_{0}^{2} / \partial \alpha$ and $\partial \delta n_{m}^{2} / \partial \alpha$, as they only result in weak self-bending of the beams, which is an effect already taken into account by the second term in Eq. (5).

It is important to distinguish between the real and imaginary parts of $\delta n_{m}^{2}$, because of the fact that when the Bragg grating inside the crystal is $\pi / 2$ phase shifted with respect to the intensity grating, the backward beam gets stronger as it travels through the crystal, while the forward beam gets depleted. In other words, the imaginary part of $\delta n_{m}^{2}$ breaks the $z \rightarrow-z$ symmetry between the forward and the backward propagating beams and induces energy transfer between them.

In Fig. 2 we show the unmodulated part $\delta n_{0}^{2}$ of the refractive index change for crystals tilted at different angles $\alpha$, calculated using Eqs. (5) and (11). It can be seen that it is the
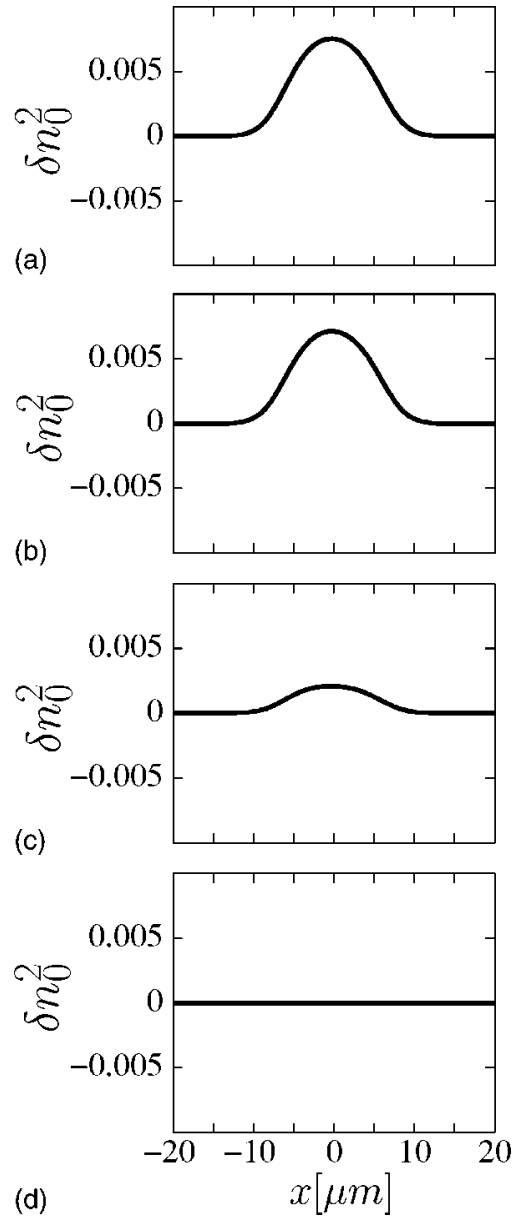

FIG. 2. Unmodulated part $\delta n_{0}^{2}$ of the refractive index change. Gaussian beams $F=B=\exp \left[-x^{2} /\left(2 \sigma^{2}\right)\right]$ with $\sigma=5 \mu \mathrm{m}$ are chosen as the beam profiles. The crystal is tilted by $\alpha=0^{\circ}$ in (a), $10^{\circ}$ in (b), $45^{\circ}$ in (c), and $90^{\circ}$ in (d). $E_{e}=3 \mathrm{kV} / \mathrm{cm}$.

strongest for $\alpha=0^{\circ}$, as should be expected. This configuration is typically employed in the investigations of spatial solitons. For $\alpha=90^{\circ}$, which is the standard configuration for experiments on pattern formation, it almost vanishes, owing to the small value of $r_{42}$.

Using Eqs. (8), (9), and (11) leads to the modulated part $\delta n_{m}^{2}$ of the refractive index change shown in Fig. 3. It can be seen that the imaginary part is dominant for tilted crystals (by a factor of about 10), i.e., the symmetry between the forward and the backward beams is broken. Furthermore, $\delta n_{m}^{2}$ is the strongest at some intermediate value of $\alpha$. Therefore, in experiments where a strong transfer of energy from one beam to the other is desired, it might be useful to tilt the crystal by $\alpha \approx 45^{\circ}$.

To confirm observations concerning the dependence of $\delta n_{m}^{2}$ on $\alpha$, we plot in Fig. 4 the amplitude and the phase of $\delta n_{m}^{2}$ at $x=0$, as functions of $\alpha$. It can be seen that the amplitude is the biggest for the value of $\alpha \approx 45^{\circ}$, and that even for small angles the phase is close to $\pi / 2$. This means that the energy transfer from one beam to the other has to be taken into account.

Thus, we can state that the counterpropagation of coherent beams in biased photorefractive crystals combines the 


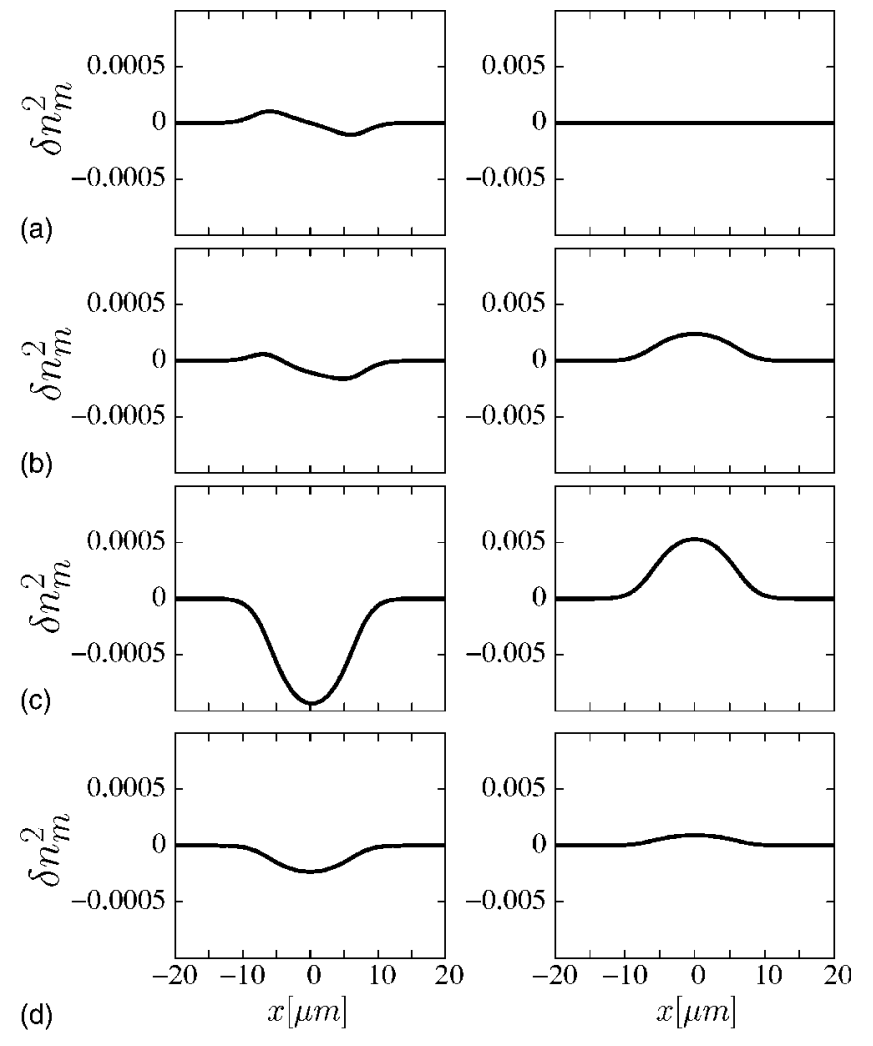

FIG. 3. Modulated part $\delta n_{m}^{2}$ of the refractive index change. The left column shows the real part, the right column the imaginary part. All parameters are as in Fig. 2.

features of both soliton formation without energy exchange and two-wave mixing in photorefractive media, namely, selffocusing by the unmodulated part $\delta n_{0}^{2}$ of the refractive index change and energy exchange by the modulated part, which is $\sim \pi / 2$ phase shifted with respect to the modulation of the light intensity.

To see whether the combination of these features leads to a propagation behavior that is a mixture of self-focusing and pattern formation, we simulated the counterpropagation of two beams with initial profiles $F=B=0.3 \exp \left[-x^{2} /\left(2 \sigma^{2}\right)\right]$

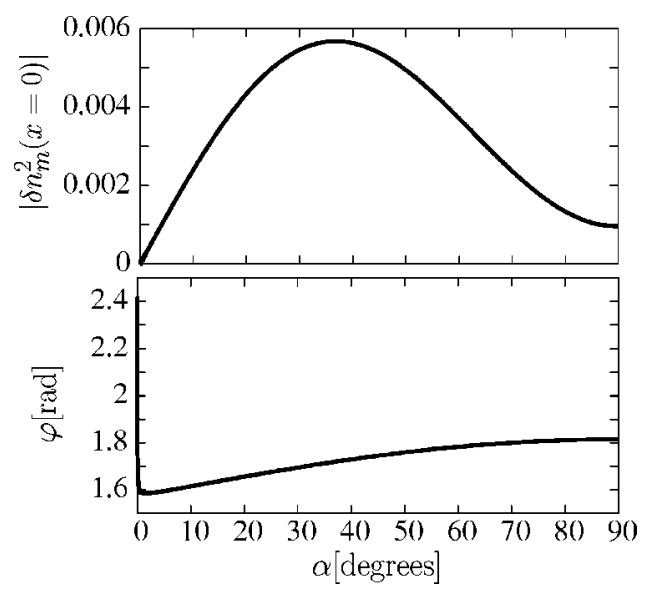

FIG. 4. Amplitude and phase of $\delta n_{m}^{2}$ at $x=0$. The upper plot shows the amplitude; the lower plot shows the phase $\varphi$, defined by $\varphi=\arctan \left[\operatorname{Im}\left\{\delta n_{m}^{2}(x=0)\right\} / \operatorname{Re}\left\{\delta n_{m}^{2}(x=0)\right\}\right]$.
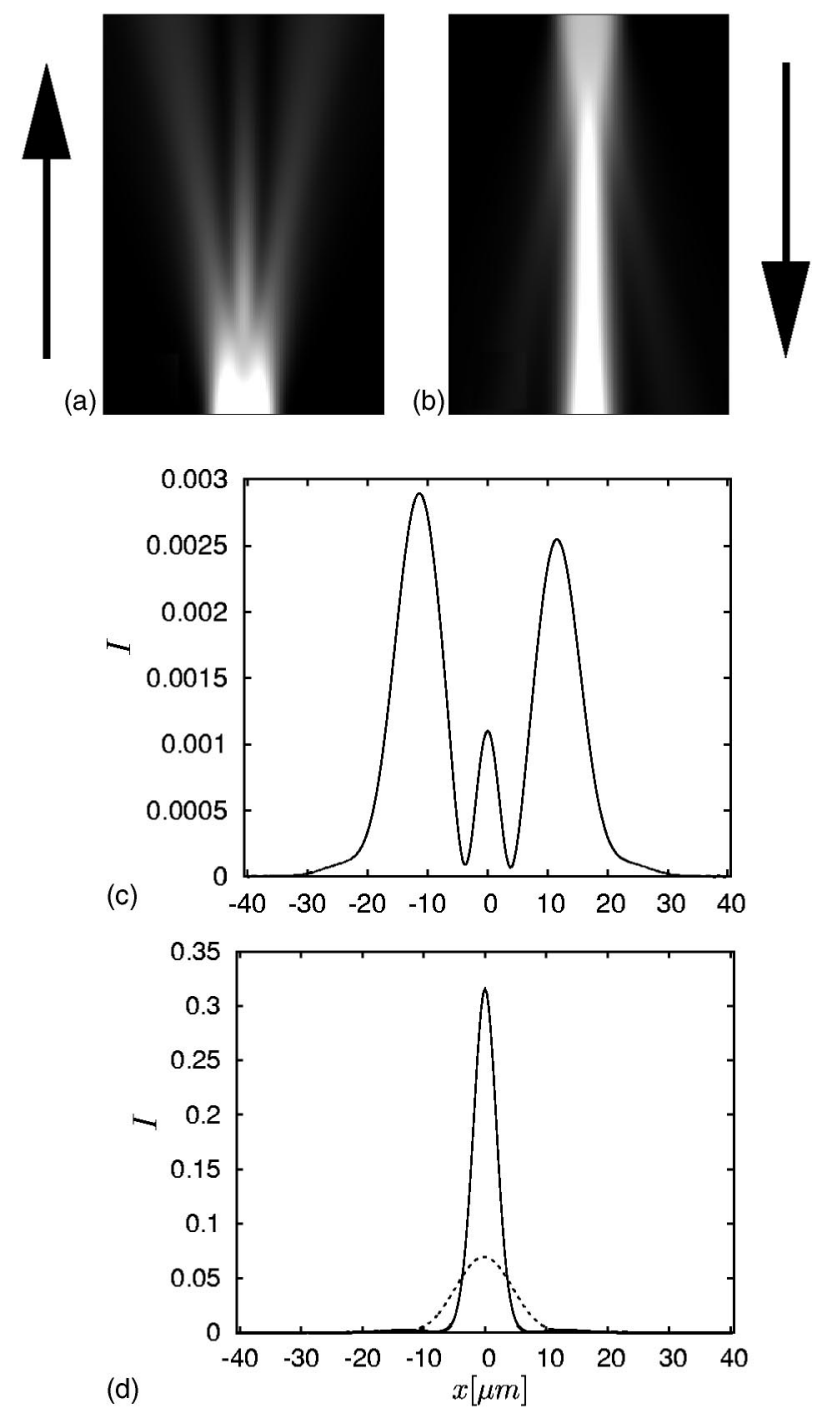

FIG. 5. Counterpropagation of two beams in a $1 \mathrm{~mm}$ long crystal. The crystal is assumed to be tilted by $\alpha=10^{\circ}$ with respect to the propagation direction. (a) shows the evolution of the forward beam (propagating from bottom to top); (b) shows the backward beam (propagating in the opposite direction). (c) shows the profile of the forward beam as it leaves the crystal. In (d) the dashed line shows the backward beam leaving the crystal after linear propagation, whereas the solid line shows it after nonlinear propagation.

with $\sigma=4 \mu \mathrm{m}$. We assumed an angle $\alpha=10^{\circ}$ and an external voltage of $E_{e}=3 \mathrm{kV} / \mathrm{cm}$. We considered a $1 \mathrm{~mm}$ thin slice of an SBN:75 crystal. The result of the simulation is presented in Fig. 5. Figures 5(a) and 5(b) show how the profiles of the beams change as they propagate. (a) shows the forward beam and (b) the backward beam, with their direction of propagation being indicated by arrows on the side of the plots. Figure 5(c) shows the profile of the forward beam as it leaves the crystal. It has split into three beams, reminiscent of the breaking of a uniform beam into stripes in experiments on pattern formation in counterpropagating beams. Finally, the solid line in Fig. 5(d) shows the backward beam as it leaves the crystal. For comparison, the dashed line shows what the beam would look like were the nonlinearity absent. One can 
see that on the one hand the backward beam gets amplified while propagating through the crystal; on the other hand the self-focusing effect of the nonlinearity is also clearly visible. For this simulation we set $T=300 \mathrm{~K}$. The effect of the selfbending is weak due to the short propagation distance. However, its effects are clearly visible in the asymmetry of the beam profile in Fig. 5(c).

In conclusion, we have found that the counterpropagation of light beams in biased crystals tilted with respect to the propagation direction of the beams induces a Bragg grating in the refractive index of the crystal, which produces a strong influence on the propagation of beams. The strength of the grating strongly depends on the angle by which the crystal is tilted, whereas its phase is always close to $\pi / 2$, except for very small angles. As a consequence, the tilted biased photorefractive crystal can exhibit a combination of features connected with soliton formation on the one hand and with pattern formation on the other hand.

The authors appreciate enlightening discussions with $\mathrm{S}$. Odoulov and C. Rotschild. M.B. and A.D. acknowledge support from the Humboldt Foundation.
[1] Y. Silberberg and I. Bar Joseph, Phys. Rev. Lett. 48, 1541 (1982).

[2] A. L. Gaeta et al., Phys. Rev. Lett. 58, 2432 (1987).

[3] M. Haelterman, A. P. Sheppard, and A. W. Snyder, Opt. Commun. 103, 145 (1993).

[4] O. Cohen et al., Phys. Rev. Lett. 89, 133901 (2002).

[5] O. Cohen et al., Opt. Lett. 27, 2013 (2002).

[6] M. Belić et al., Phys. Rev. E 68, 025601 (2003).

[7] E. DelRe, A. Ciattoni, B. Crosignani, and P. DiPorto, J. Nonlinear Opt. Phys. Mater. 8, 1 (1999).
[8] For an overview see Opt. Photonics News 13(2) (2002), special issue on solitons, edited by M. Segev.

[9] D. N. Christodoulides and M. I. Carvalho, J. Opt. Soc. Am. B 12, 1628 (1995).

[10] P. Yeh, Introduction to Photorefractive Nonlinear Optics (Wiley, New York, 1993).

[11] A. A. Zozulya and D. Z. Anderson, Phys. Rev. A 51, 1520 (1995).

[12] C. Rotschild, O. Cohen, O. Manela, T. Carmon, and M. Segev, J. Opt. Soc. Am. B 21, 1354 (2004). 